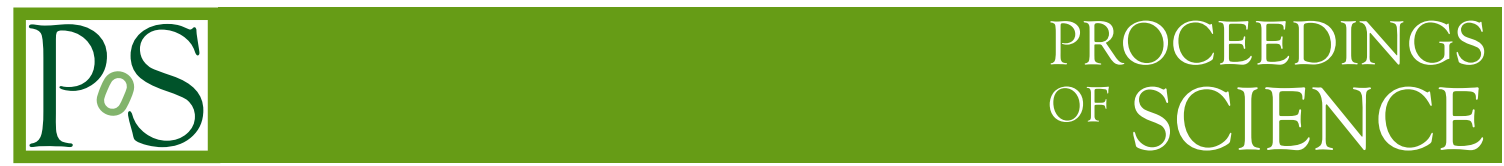

\title{
Recent Results in Single Top Quark Production at the LHC
}

\author{
Galo Gonzalvo Rodriguez*, on behalf of the ATLAS and CMS Collaborations \\ Instituto de Física Corpuscular, \\ Catedrático José Beltrán 2, Valencia, Spain \\ E-mail: galo.rafael.gonzalvo.rodriguez@cern.ch
}

\begin{abstract}
This document presents the most recent results in the field of single-top-quark production using data recorded by both the ATLAS and CMS detectors at the LHC. Both differential and inclusive crosssections measurements are reported for the main production modes. In addition, the observation by ATLAS of the exotic $t Z q$ production mode is also described. This document concludes with the first measurement of the full top quark and antiquark polarisation vectors performed by the ATLAS collaboration. The results are compared with theoretical predictions, and no significant deviations from the Standard Model are observed.
\end{abstract}

The Ninth Annual Conference on Large Hadron Collider Physics - LHCP2021

7-12 June 2021

Online

${ }^{*}$ Speaker 


\section{Introduction}

At the LHC top quarks can be produced both in pairs via the strong interaction or singly via the electroweak interaction. Three processes contribute to single-top-quark production at leading order (LO): the $t$-channel, the $s$-channel and the production of a $W$ boson in association with a top quark ( $t W$-channel). All these processes make possible to probe the $t W b$ vertex and are sensitive to the Cabibbo-Kobayashi-Maskawa (CKM) matrix element $V_{t b}$. In addition, top quarks are produced polarised only in single-top-quark events. A review of the most recent results in the field of singletop-quark production from both the ATLAS [1] and the CMS [2] collaborations is given in the following.

\section{Differential cross-section measurements}

Differential cross-section measurements can contribute to constraining the effective field theory $(\mathrm{EFT})^{1}$ operators [3], the top quark mass, the renormalisation and factorisation scales, and the parton distribution functions (PDFs) of the proton [4].

The CMS collaboration has recently measured the $t(t W)$-channel differential cross-section in the lepton (dilepton) channel using $36 \mathrm{fb}^{-1}$ of data collected by the CMS detector at a center-of-mass energy $\sqrt{s}=13 \mathrm{TeV}[5,6]$. In the $t$-channel analysis, the results are unfolded to both the particle and the parton levels and compared with theoretical predictions. In addition, differential ratios of the top quark production rates to the sum of the top quark and antiquark rates are also provided, which are sensitive to the ratio of the up to down quark content of the proton [7,8]. The spin asymmetry $A_{l}$, which is intimately related with the top quark polarisation, is also provided. The obtained result $A_{l}=0.440 \pm 0.070$ is compatible with the SM prediction $A_{l}^{S M}=0.436$, with negligible uncertainty.

In the $t W$ analysis, the results are unfolded to the particle level and the measurements are compared with theoretical predictions. Figure 1 shows the differential cross-section measurements for both the $t$-channel (left) and the $t W$-channel (right) analyses compared to different Monte Carlo (MC) generators.

\section{Inclusive measurements}

Recent results from both the ATLAS and CMS collaborations have provided a measurement for the first time of the inclusive $t W$-channel production cross-section in the lepton+jets channel $[9,10]$. ATLAS uses $20.2 \mathrm{fb}^{-1}$ of data recorded at $\sqrt{s}=8 \mathrm{TeV}$, whereas CMS uses $36 \mathrm{fb}^{-1}$ recorded at $\sqrt{s}=13 \mathrm{TeV}$. The results by ATLAS of $\sigma_{t W}=26 \pm 7$ (syst. + stat.) pb are compatible with the SM predictions at NLO at $\sqrt{s}=8 \mathrm{TeV}, \sigma_{t W}^{S M}=22.4 \pm 1.5 \mathrm{pb}$. Similarly, the results by CMS of $\sigma_{t W}=89 \pm 4$ (stat.) \pm 12 (syst.) pb are compatible with the SM predictions at NLO at $\sqrt{s}=13 \mathrm{TeV}$, $\sigma_{t W}^{S M}=71.7 \pm 3.8 \mathrm{pb}$

\section{4. $t Z q$ observation}

At NLO, the production of top quarks in the $t$-channel with additional radiation of a $Z$ boson from any quark or the $W$ boson propagator leads to the $t Z q$ production mode. This channel allows

\footnotetext{
${ }^{1} \mathrm{EFT}$ is a model independent way to parametrise possible deviations from the SM predictions.
} 

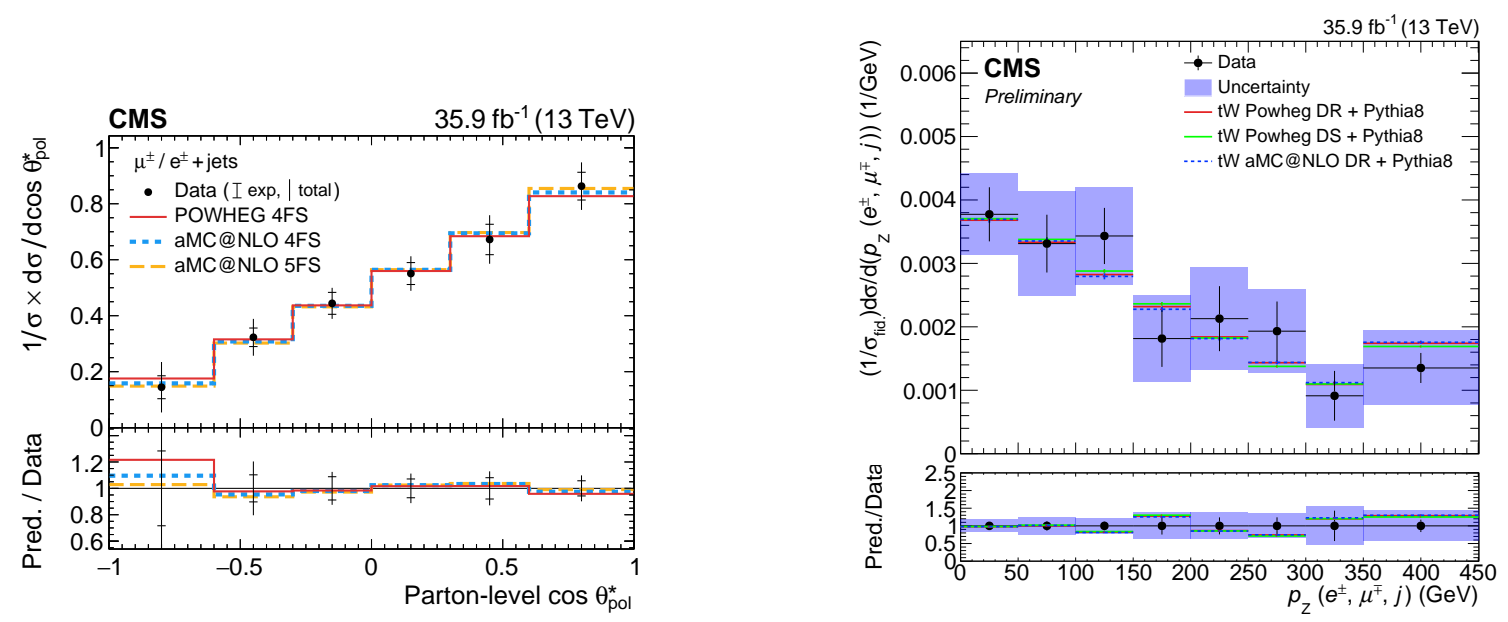

Figure 1: (a): Normalised differential cross section for the $t$-channel production at the parton level as a function of the cosine of the top quark polarisation angle. The total uncertainty is indicated by the vertical lines, while horizontal bars indicate the statistical and experimental uncertainties. Three different predictions from event generators are shown by the solid, dashed, and dotted lines. The lower panel shows the ratios of the predictions to the data [5]. (b): Normalized differential $t W$ production cross section as a function of the longitudinal momentum of the muon, electron and jet system. The solid band represents the total uncertainty. Predictions from MC generators are also shown. In the bottom panel, the ratio between predictions and data is shown [6].

to study the $t Z$ and $W Z$ couplings. In addition, the NLO corrections are small, and the deviations can be interpreted within EFT to check for possible deviations from the SM predictions. Latest results by ATLAS [11] have reported an observation of the $t Z q$ production mode. The analysis uses $139 \mathrm{fb}^{-1}$ collected at $\sqrt{s}=13 \mathrm{TeV}$. The obtained result of $\sigma_{t Z q}=97 \pm 13$ (stat.) \pm 7 (syst.) fb is compatible with the SM predictions, $\sigma_{t Z q}^{S M}=102_{-2}^{+5} \mathrm{fb}$, and with the previous result by CMS of $\sigma_{t Z q}=111 \pm 13$ (stat. $)_{-9}^{+11}$ (syst.) fb [12].

\section{Top quark polarisation}

At the LO in the $t$-channel single top quarks are produced with their spin aligned along the direction of the down-type quarks. Latest measurements by the ATLAS collaboration [13] using the leptonic decay of the top quark assess its polarisation using the angular distributions of its decay products in the top-quark rest frame. The analysis uses $139 \mathrm{fb}^{-1}$ of data collected by the ATLAS detector at $\sqrt{s}=13 \mathrm{TeV}$.

A profile likelihood fit is applied to extract the polarisation vectors using fully polarised templates as provided by the Pвотоs generator [14]. The obtained results are displayed in Table 1. These are compatible with the SM MC predictions: $P_{x^{\prime}}^{t}=0.040 \pm 0.012, P_{x^{\prime}}^{\bar{t}}=-0.070 \pm 0.016$, $P_{z^{\prime}}^{t}=1.024 \pm 0.015, P_{z^{\prime}}^{t}=-0.967 \pm 0.020\left(P_{y^{\prime}}\right.$ is expected to be 0 from charge conjugation parity (CP) symmetry).

In addition, this analysis also provides normalised differential cross-section measurements as a function of the polarisation angles (see Figure 2). The distributions are unfolded to the particle level and the measurements are performed in a fiducial region. These results are interpreted in an 


\begin{tabular}{lcc}
\hline \hline Parameter & Extracted value & (stat.) \\
\hline$P_{x^{\prime}}^{t}$ & $+0.01 \pm 0.18$ & $( \pm 0.02)$ \\
$P_{x^{\prime}}^{\bar{t}}$ & $-0.02 \pm 0.20$ & $( \pm 0.03)$ \\
$P_{y^{\prime}}^{t}$ & $-0.029 \pm 0.027$ & $( \pm 0.011)$ \\
$P_{y^{\prime}}^{\bar{t}}$ & $-0.007 \pm 0.051$ & $( \pm 0.017)$ \\
$P_{z^{\prime}}^{t}$ & $+0.91 \pm 0.10$ & $( \pm 0.02)$ \\
$P_{z^{\prime}}^{\bar{t}}$ & $-0.79 \pm 0.16$ & $( \pm 0.03)$ \\
\hline \hline
\end{tabular}

Table 1: Polarisation values as extracted from data, including total and statistical-only uncertainties in the fit [13].

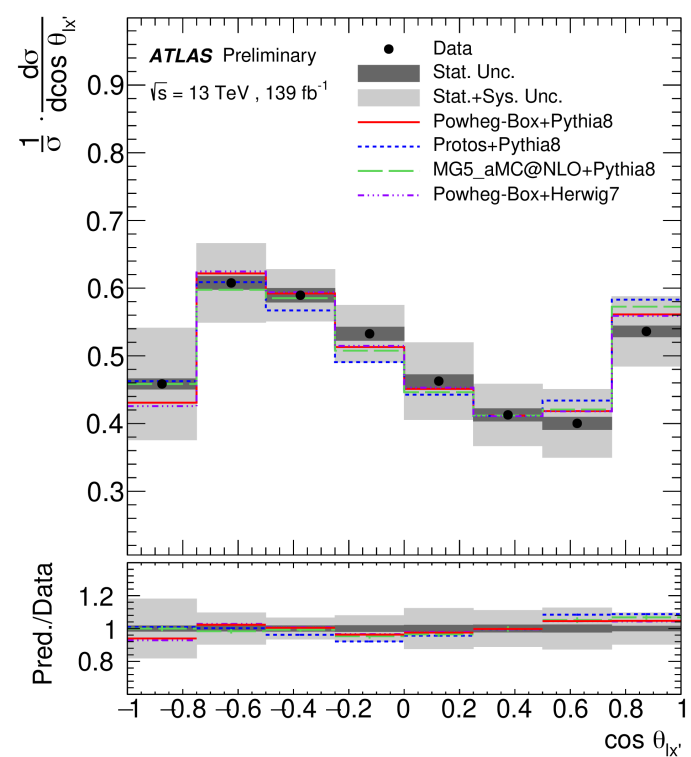

Figure 2: Particle-level normalised differential cross-section as a function of the $\cos \theta_{l x^{\prime}}$ angular variable along with various SM MC predictions of the $t$-channel signal for both top quarks and top antiquarks. The data, shown as the black points with statistical uncertainties, are compared to predictions (lines) using different MC generators. The uncertainty bands include both the statistical and systematic uncertainties. The lower panels show the ratio of prediction to data in each bin [13].

EFT context to set limits on the real part $C_{t W} \epsilon[-0.7,1.5]$ and the imaginary part $C_{i t W} \epsilon[-0.7,0.2]$ of the dipole operator Wilson coefficient at $95 \%$ confidence level. The limits on the imaginary part are the best existing limits from high-energy experiments.

\section{Summary}

Single-top-quark production is an important part of the physics program at the LHC. It provides a unique room to investigate the electroweak interaction and the physics of the $t W b$ vertex. The ATLAS and CMS collaborations have contributed to this field through many exciting new results. So far all the reported measurements are consistent with the SM predictions. 


\section{References}

[1] ATLAS Collaboration, 2008 JINST 3 S08003

[2] CMS Collaboration, 2008 JINST 3 S08004

[3] N. P. Hartland et al., JHEP 04 (2019) 100

[4] P. Kant et al., Comput. Phys. Commun. 191 (2015) 74-89

[5] CMS Collaboration, Eur. Phys. J. C 80 (2020) 370

[6] CMS Collaboration, CMS-PAS-TOP-19-003, https://cds.cern.ch/record/2712818

[7] E. L. Berger et al., Phys.Rev.D 94 (2016) 7, 071501

[8] S. Alekhin, et al., Phys.Rev.D 96 (2017) 1, 014011

[9] ATLAS Collaboration, Eur. Phys. J. C 81 (2021) 8, 720

[10] CMS Collaboration, CMS-PAS-TOP-20-002, https://cds.cern.ch/record/2759300, arxiv:2109.01706

[11] ATLAS Collaboration, JHEP 07 (2020) 124

[12] CMS Collaboration, Phys. Rev. Lett. 122, 132003 (2019)

[13] ATLAS Collaboration, ATLAS-CONF-2021-027, http://cdsweb.cern.ch/record/2773738

[14] J.A. Aguilar-Saavedra, Nucl. Phys. B 804 (2008) 160-192 